\title{
Study of self-shadowing effect as a simple means to realize nanostructured thin films and layers with special attentions to birefringent obliquely deposited thin films and photo-luminescent porous silicon
}

\section{Tomoyoshi Motohiro}

Tomoyoshi Motohiro, "Study of self-shadowing effect as a simple means to realize nanostructured thin films and layers with special attentions to birefringent obliquely deposited thin films and photo-luminescent porous silicon," Proc. SPIE 10731, Nanostructured Thin Films XI, 1073102 (7 September 2018); doi: 10.1117/12.2322702

Event: SPIE Nanoscience + Engineering, 2018, San Diego, California, United States 


\title{
Study of self-shadowing effect as a simple means to realize nanostructured thin films and layers with special attentions to birefringent obliquely deposited thin films and photo-luminescent porous silicon
}

\author{
Tomoyoshi Motohiro*a,b \\ aToyota Central R\&D Labs., Inc., Nagakute, Aichi, Japan, 480-1192; \\ ${ }^{\mathrm{b}}$ Green Mobility Research Institute, Institutes of Innovation for Future Society, Nagoya University, \\ Furo-cho, Chikusa-ku, Nagoya, Japan,464-8603
}

\begin{abstract}
Self-shadowing effect is elucidated as a simple means without "nanotechnology" to realize nanostructured thin films and layers in two typical prominent nanostructure effects, that is, birefringence of obliquely deposited thin films and intense visible photoluminescence of porous $\mathrm{Si}$. In oblique deposition, the self-shadowing effect takes place in ballistic deposition and accumulation of materials on a vacuum / substrate interface. In porous $\mathrm{Si}$, the drift diffusion of positive holes from an anode thorough $\mathrm{Si}$ to $\mathrm{Si} / \mathrm{HF}$ solution interface causes a modified diffusion limited aggregation of positive holes which leads directly to formation of pores.
\end{abstract}

Keywords: self-shadowing effect, oblique deposition, porous silicon, birefringence, visible photoluminescence, nanostructured thin film, diffusion limited aggregation, thin film retardation plate

\section{INTRODUCTION}

We sometimes encounter "nanostructures" without intention to form them in daily experiments with rather simple macroscopic experimental settings. The very similar four SEM photographs of ripple structures with different scales in Figs 1 show evolution of surface morphology of a transparent amorphous fused silica with elapsed time under oblique
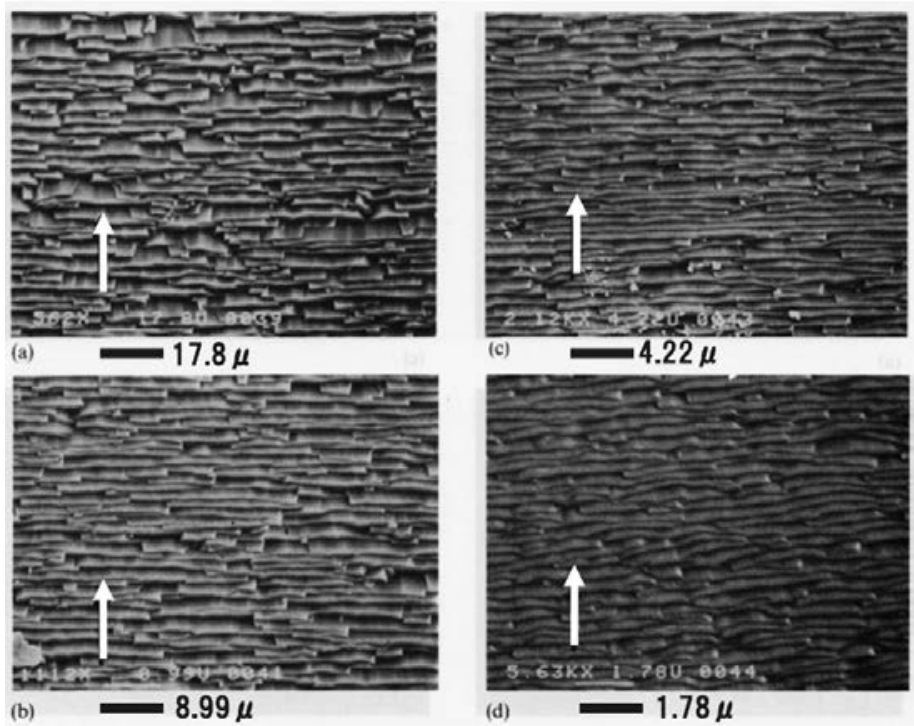

Figure 1 Spontaneous formation and evolution of ripple structures by $45^{\circ}$ oblique $4.25 \mathrm{keV}$ Ar ion beam bombardment of the surface of transparent fused silica. SEM photographs shows the surface morphologies at different ion doses: (a) $0.36 \mathrm{C} / \mathrm{cm}^{2}$, (b) $0.23 \mathrm{C} / \mathrm{cm}^{2}$, (c) $0.14 \mathrm{C} / \mathrm{cm}^{2}$, (d) $0.09 \mathrm{C} / \mathrm{cm}^{2}$. 
4.25 $\mathrm{keV}$ Ar ion bombardment ${ }^{1}$. Although the array of ripple structure looks rather random, their average period increased with $1.88^{\text {th }}$ power of the ion dose $\mathrm{e}^{2}$. In Fig.1(d) at the ion dose of $0.09 \mathrm{C} / \mathrm{cm}^{2}$, the average wavelength was around $400 \mathrm{~nm}$, and it is conjectured that the ripple formation started with much smaller scale with much less ion dose, and quite controllable by ion dose. The ripple structures are composed mainly of two planes inclined at $-19^{\circ}$ and $85^{\circ}$ from the incident angle of argon beam at $45^{\circ}$. The former is near the angle of maximum in the angular dependence of sputtering yield, showing that erosion by sputter etching is a major promoting event for ripple formation. Oblique sputter etching of $-19^{\circ}$ planes naturally causes preferential forward ejection of sputtered atoms. Redeposition of these sputtered atoms is a kind of negative feedback of the erosion by sputter etching. The second plane inclined $85^{\circ}$ from the incidence of Ar ion beam might have grown effectively by the redeposition of sputtered particles. As a balance of the promoting event: sputter-etching and negative feedback event: redeposition, it is conjectured that the ripple structures emerged and evolved spontaneously. This is quite similar to the spontaneous formation of sand ripples by wind, which is formed in the balance of blowing off of the sand particles and their redeposition ${ }^{2}$. The incident angles of $\mathrm{Ar}$ ions of $22.5^{\circ}$ and $67.5^{\circ}$ did not gave such ripple structures. The rather simple macroscopic experimental setting, that is, the angular setting of $45^{\circ}$ incidence of $\mathrm{Ar}$ ion beam in this case is advantageous for applications, such as, "nanostructures in a wide area at low cost", in connection to optical grating, liquid crystal alignment, anisotropic water repellant effect,..etc. The number of annual publications on the ripple formation by ion beam sputter etching increased with increased attention to nanotechnology typically after $1994^{2}$. Self-shadowing which is the main topic in this paper, is another typical effect in the same taste with the spontaneous ripple formation by oblique ion beam etching mentioned above. With rather simple macroscopic experimental settings, i.e., oblique deposition and electrochemical anodization, we could experience visual observation of effects of nanostructure origin far before 1994, i.e., birefringence from large area amorphous oxide thin films and visible intense photoluminescence from entire surface of $101.6 \mathrm{~mm}$ diameter wafers of Si which is inherently non-luminescent because of its electronic structure of indirect transition nature.

\section{SELF-SHADOWING IN OBLIQUE DEPOSITION}

\subsection{Geometrical features of nanostructures by self-shadowing in oblique deposition elucidated by computer modeling}

Computer modeling has been a useful means to elucidate geometrical features of obliquely deposited nanostructured thin films $^{3-5}$. Figure 2 shows a method of a three dimensional computer modeling ${ }^{6}$. Using pairs of random numbers, particles are supposed to be homogeneously generated on the plane of particle generation whose surface normal $\mathbf{n}$ is inclined from the surface normal $\mathrm{Z}$ of the plane of the substrate surface by a deposition angle $\delta$. The generated particles are supposed to drift in parallel with $\mathbf{n}$ until they make their first contacts with the substrate plane or previously deposited particles. The diameter of the particles is taken as a unit of $\mathrm{X}, \mathrm{Y}$, and $\mathrm{Z}$ axes. Particles are supposed to freeze at their rest points and no further relaxation processes are considered so as to elucidate genuine nature of self-shadowing effect. Figure 3

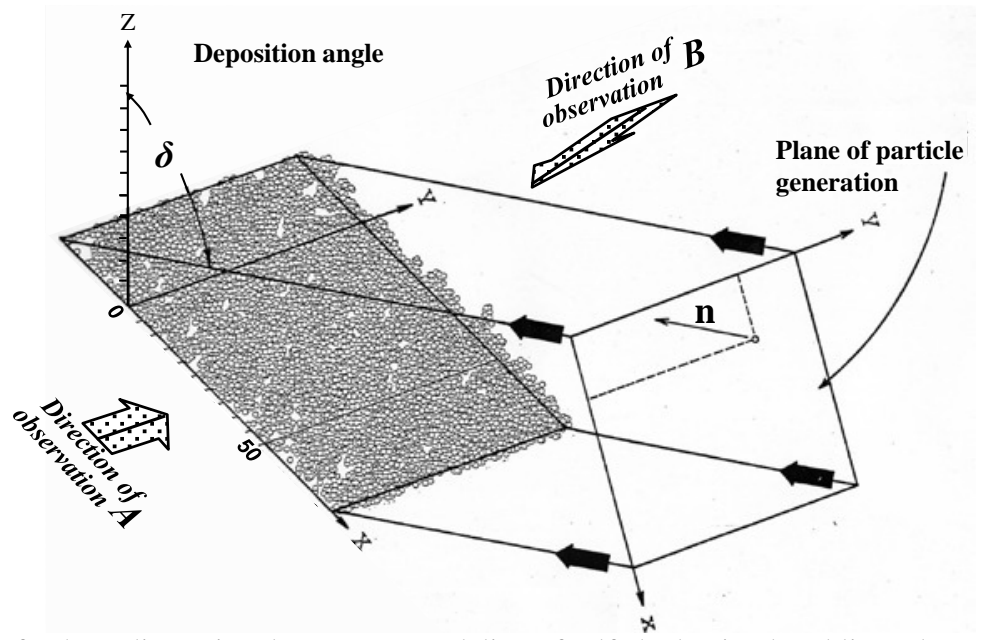

Figure 2 Geometry of a three dimensional computer modeling of self-shadowing by oblique deposition. 


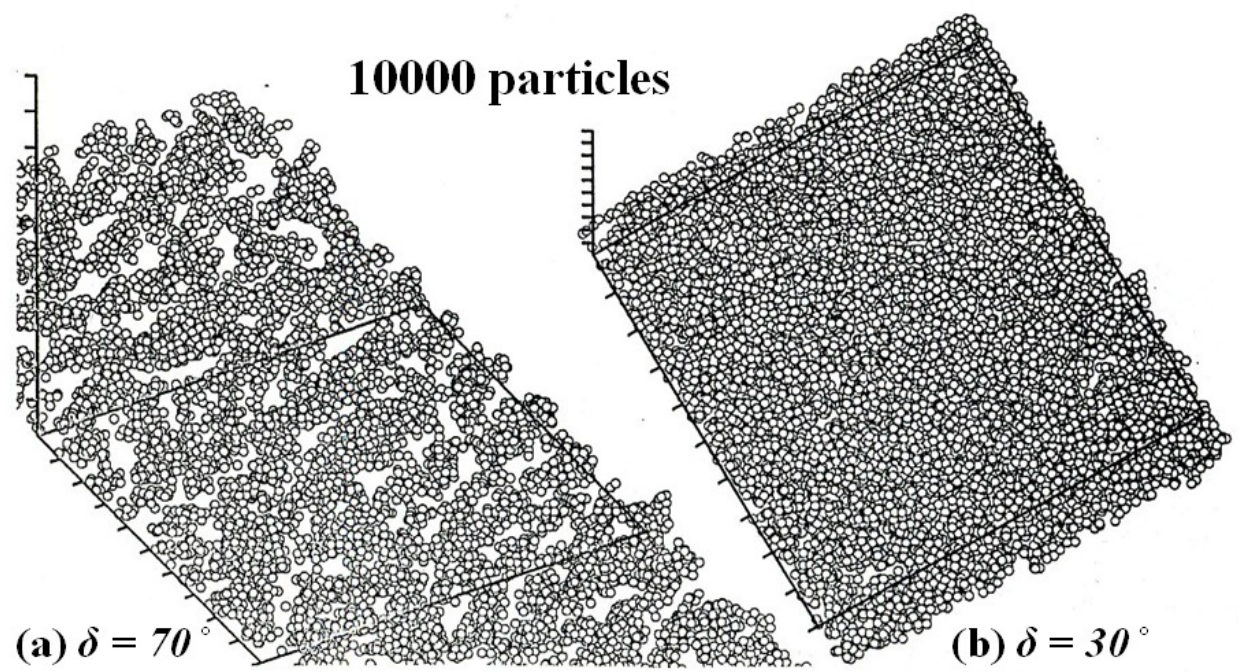

Figure 3 Morphology of the obliquely deposited 10000 particles at (a) $\delta=70^{\circ}$ and (b) $\delta=30^{\circ}$.

shows distribution of 10000 particles obliquely deposited at (a) $\delta=70^{\circ}$ and (b) $\delta=30^{\circ}$. Self-shadowing has caused formation of many voids between clusters of deposited particles in the case of (a) $\delta=70^{\circ}$, while a relatively densely packed distribution of particles without remarkable voids was reproduced in the case of (b) $\delta=30^{\circ}$. Observation from lateral direction $\mathbf{A}$ indicated in Fig.2 gives another view of this self-shadowing structure as shown in Fig.4. Figure 4 clearly reproduced the well-known growth of columnar structures toward the direction much less than deposition angle: $85^{\circ}$ measured from the substrate surface normal. The columnar structures shadow the voids behind the columns so as not to fill them with incoming particles at the incident angle of $85^{\circ}$. Observation from the direction B indicated in Fig.1, looking down the substrate, elucidates different feature of the self-shadowing structure. Figure 5 shows the same

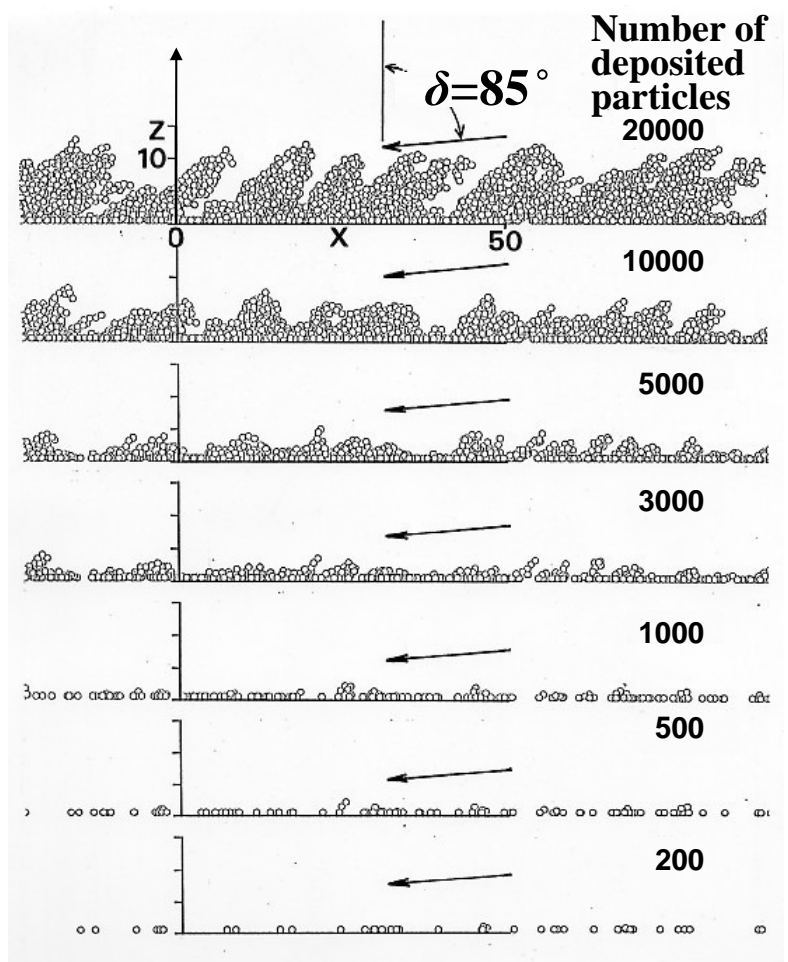

Figure 4 Observation of growth of self-shadowing structures by oblique deposition at $\delta=85^{\circ}$ from the direction perpendicular to the direction of particle incidence and the substrate surface normal. 


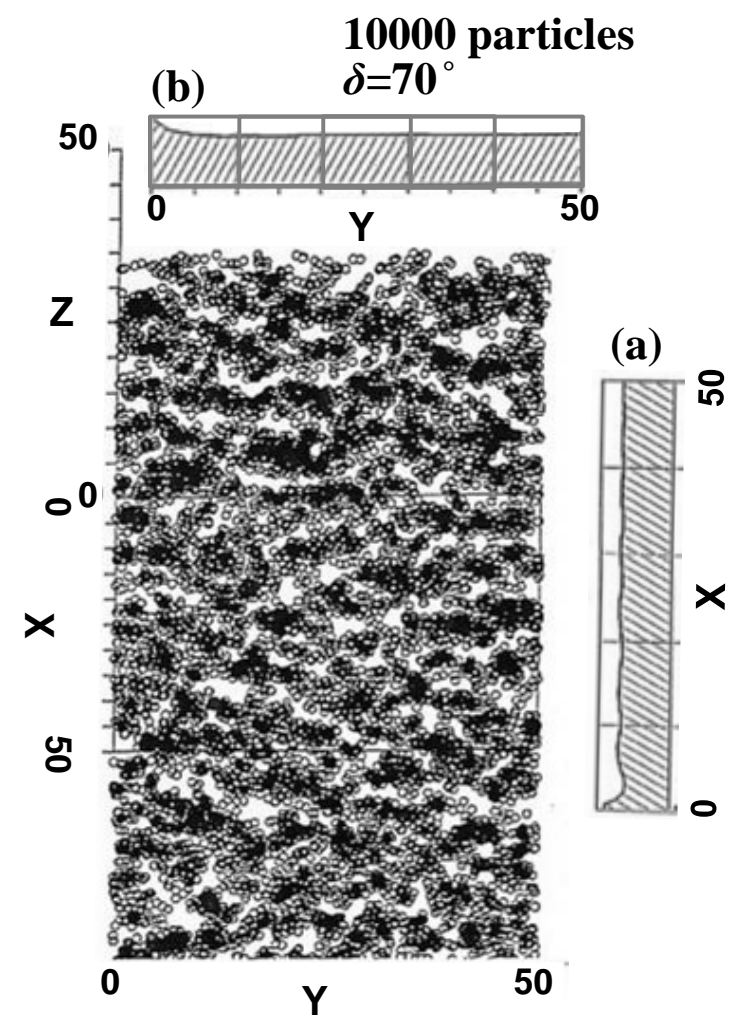

Figure 5 the distribution of 10000 particles obliquely deposited at $\delta=70^{\circ}$, 'fluoroscoped' from the direction at $54^{\circ}$ from the substrate normal (z-axis). The attached two hatched graphs (a) and (b) show the distributions of the length of the vectors connecting arbitrary pairs of projected particles in the main figure, (a) for those vectors whose angular deviations from X-direction and (b) from Y-direction are within $\mp 9^{\circ}$, respectively

distribution of 10000 particles obliquely deposited at $\boldsymbol{\delta}=70^{\circ}$ as Fig.3, but 'fluoroscoped' from the direction B which is inclined at $54^{\circ}$ from the substrate normal (Z-axis). There exists an anisotropic distribution of particles, being more likely to be connected toward the direction in parallel with $\mathrm{Y}$-axis rather than the direction in parallel with $\mathrm{X}$-axis. The attached two hatched graphs (a) and (b) show the distributions of the length of the vectors connecting the centers of arbitrary pairs of projected particles in the main figure, (a) for those vectors whose angular deviations were within $\mp 9^{\circ}$ from X-direction (a) and from Y-direction (b), respectively. The existence of the depleted zone only in the graph (a) elucidates that particles are more likely connected toward Y direction. This anisotropic connectivity of particles could be found in the case of $\boldsymbol{\delta}$ over $30^{\circ}$, indicating that oblique deposition makes inclined quasi-layerd structure of alternating high density and low density layers. Alternative appearance of clusters of particles (columns) and voids along the X direction is the origin of this anisotropic connectivity. Figure 6 shows a lateral array of longitudinal stripes in which black portions and white portions appears alternatively. An anisotropic connectivity of black portions towards horizontal direction or quasilayered structure can be observed, reproducing the feature of the anisotropic connectivity shown in Fig.5. As typically shown in Fig.4 and 5, the three dimensional modeling of oblique deposition elucidated biaxial nature of the obliquely deposited thin film irrespective of the crystallinity of the film substance. The next subsection experimentally elucidates this biaxial nature of obliquely deposited oxide films.

\subsection{Oblique deposition of oxide thin films and their biaxial nature}

Figure 7 schematically shows an experimental apparatus specially designed for studies of oblique deposition ${ }^{7}$. By means of a rotatable duralmin flange of $600 \mathrm{~mm}$ in diameter on which the entire substrate holder system rests including heater unit, slit to limit deposition angle, and motor-driven substrate sliding motion system, the deposition angle $\boldsymbol{\delta}$ can be set at any angle between $0^{\circ}$ (normal incidence) and $90^{\circ}$. The evaporation source with four hearths on a revolver (ULVAC Corporation) was employed. Film thickness and deposition rate were monitored with an Inficon crystal thickness monitor calibrated with step height measurements at the edge of the evaporation mask by a stylus method. A cryopump unit 


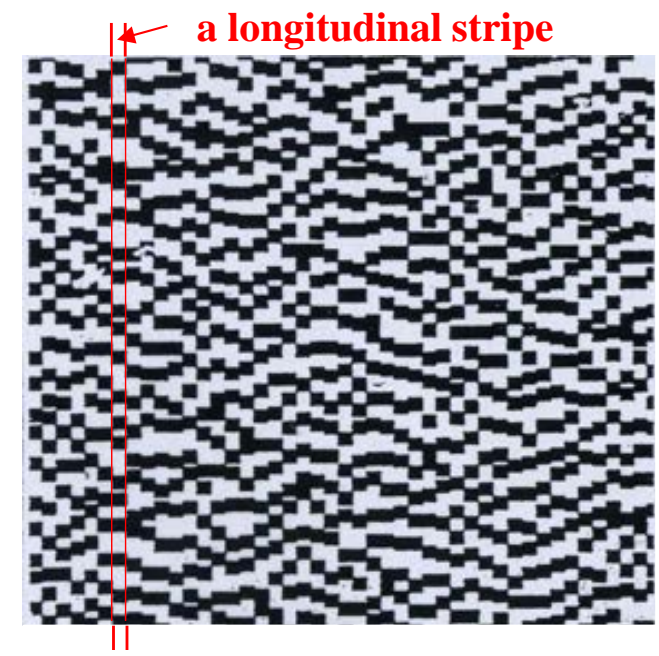

Figure 6 a lateral array of longitudinal stripes in which black portions and white portions appears quasi-periodically reproduces the anisotropic connectivity of particles in Fig.5.

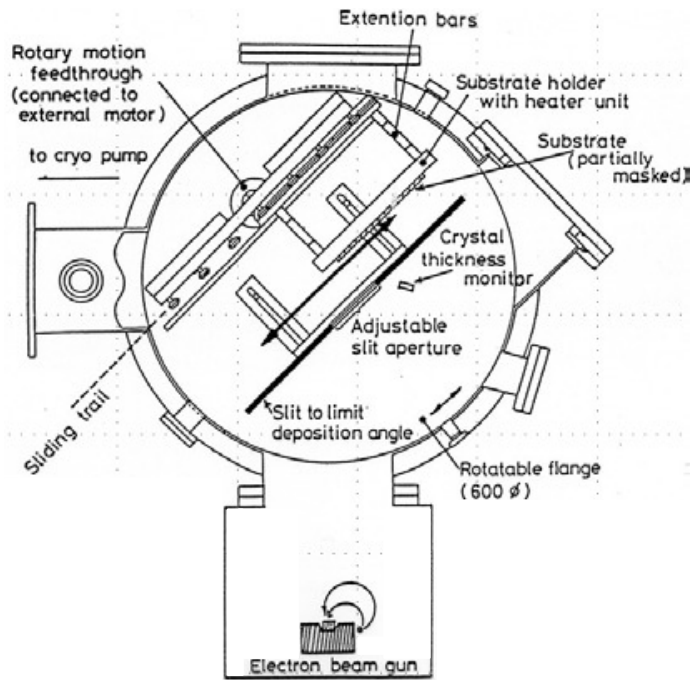

Figure 7 Schematic drawing of the experimental apparatus specially designed for studies on oblique deposition

(Aisin Seiki Co.) of $203.2 \mathrm{~mm}$ in diameter was employed for the evacuation system. Although the ultimate pressure of the system was typically $6.7 \times 10^{-5} \mathrm{~Pa}$, depositions were started when the system was pumped down below $2.7 \times 10^{-4} \mathrm{~Pa}$. Pellets or grains of pure inorganic oxides of $3 \mathrm{~N}$ grade (High Purity Chemetals Co.) were used as the source materials for evaporation. The substrates used were CG7059 or commercially available floating glass plates.

Figure 8 (a) shows a typical example of a cross-sectional SEM image of an obliquely deposited $\mathrm{WO}_{3}$ film at $\delta=71.9^{\circ}$ and at room temperature ${ }^{8}$. The inclination angle of the columnar structures: $\alpha$ measured from the substrate normal, is much less than $\delta$ as shown in Fig.4. Fig.8 (b) shows the relation between $\alpha$ and $\delta$ for $\mathrm{WO}_{3}$ films and (c) for $\mathrm{Ta}_{2} \mathrm{O}_{5}$ films ${ }^{7}$. It is shown that $\alpha$ increases linearly with $\delta$, but the gradient depends on materials. This may be caused by the material difference of mobility of adatoms after the deposition in contrast to the frozen model in Fig.4. Using the three of 4 hearths in the apparatus shown in Fig.7, an $1.4 \mu \mathrm{m}$ obliquely deposited $\mathrm{WO}_{3}$ film, an $0.5 \mu \mathrm{m}$ normally deposited $\mathrm{SiO}_{2}$ film and an $0.28 \mu \mathrm{m}$ normally deposited $\mathrm{Al}$ film were stacked successively in vacuum ${ }^{8}$. After the stacked sample was brought out from the vacuum chamber into the air, many tiny elliptical blue patches emerged and grew right before our eyes with their major axes perpendicular to the azimuthal direction of vapor incidence as shown in Fig.9. The ratio of the length of the minor axis $\boldsymbol{b}$ to that of the major axis $\boldsymbol{a}$ of the ellipse decreased with $\boldsymbol{\delta}$ as shown in the rightmost graph in Fig.9. Since the whole area of the sample was homogeneously colored blue when we did not insert the $\mathrm{SiO}_{2}$ film between 


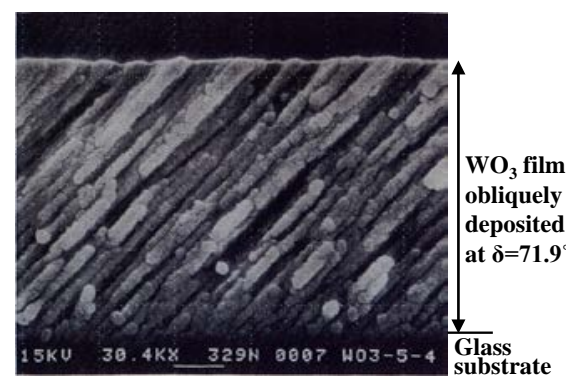

(a) SEM image of $\mathrm{WO}_{3}$ film

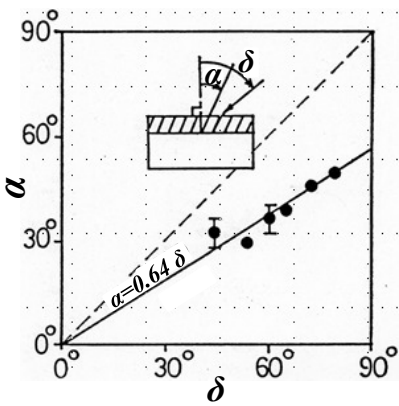

(b) $\alpha-\delta$ relation in $\mathrm{WO}_{3}$

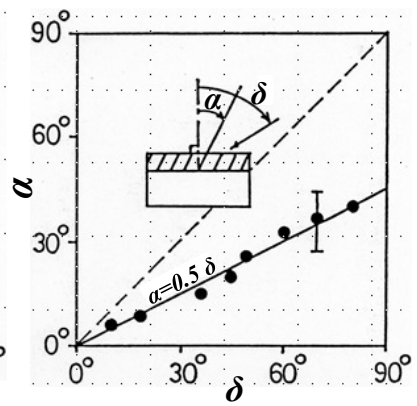

(c) $\alpha$ - $\delta$ relation in $\mathrm{Ta}_{2} \mathrm{O}_{5}$

Figure 8 Cross-sectional SEM image of an obliquely deposited $\mathrm{WO}_{3}$ film:(a), a relation between the inclination angle of columnar structures $\alpha$ and the angle of oblique deposition $\delta$ in the case of $\mathrm{WO}_{3}$ film:(b) and $\mathrm{Ta}_{2} \mathrm{O}_{5}$ film:(c).

the obliquely deposited $\mathrm{WO}_{3}$ film and the $\mathrm{Al}$ film, something came from the Al film through pinholes in the $\mathrm{SiO}_{2}$ film and diffused into $\mathrm{WO}_{3}$ film and colored $\mathrm{WO}_{3}$ film blue in the case of Fig.9. Taking the fact that the emergence of elliptical blue patches started just after air exposure, and high rate of diffusion in the $\mathrm{WO}_{3}$ film, the 'something' can be $\mathrm{H}^{+}$dissociated from water vapor chemically adsorbed on Al. Incorporation of $\mathrm{H}^{+}$ions into $\mathrm{WO}_{3}$ film could cause color change into blue just as in electrochromic displays. The growth of elliptical blue patches indicates anisotropic diffusion of $\mathrm{H}^{+}$ions. The fact that the ratio $\boldsymbol{b} / \boldsymbol{a}$ decreases rapidly with $\boldsymbol{\delta}$ in the rightmost graph in Fig.9 teaches that the anisotropic connectivity as shown in Fig. 5 and 6 was realized in the obliquely deposited $\mathrm{WO}_{3}$ film, and diffusion of $\mathrm{H}^{+}$ions was enhanced toward the direction perpendicular to the azimuthal direction of vapor incidence by anisotropic connectivity.
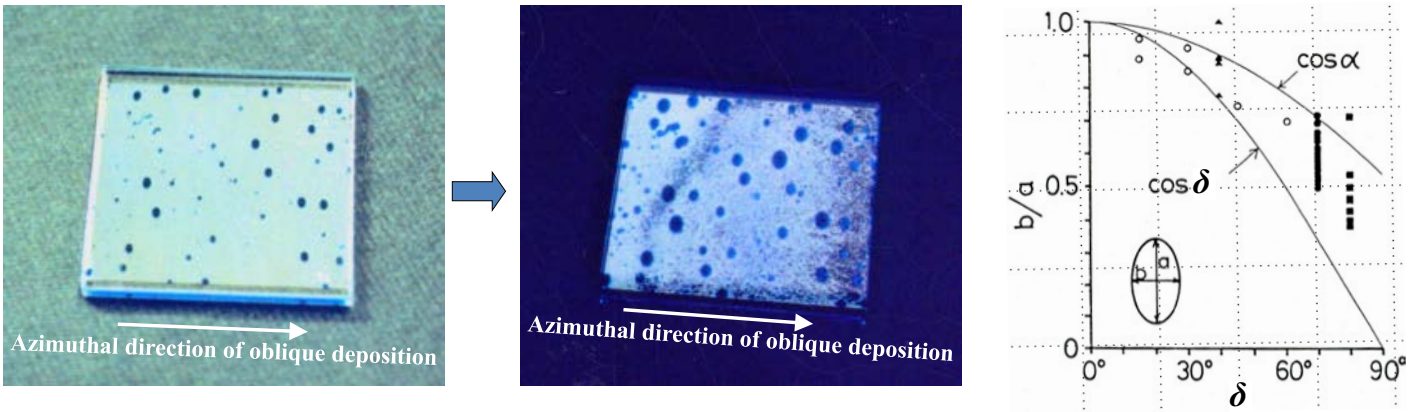

Figure 9 Growth of elliptical blue patches observed through the glass substrate right before our eyes just after the air exposure of a sample composed of a glass substrate / an obliquely deposited $\mathrm{WO}_{3}$ film / a normally deposited $\mathrm{SiO}_{2}$ film / a normally deposited Al film. The ratio of the length of the minor axis $\boldsymbol{b}$ to the that of the major axis $\boldsymbol{a}$ of the ellipse changes with $\boldsymbol{\delta}$ as shown in the rightmost graph.

Fig.8 and Fig.9 experimentally elucidated the biaxial nature of obliquely deposited oxide films in addition to the simulated results shown in Fig. 4 and Fig. $5^{9}$. Although the most of obliquely deposited oxides at room temperature are amorphous, the biaxial nature gives an optical property of birefringence which can be applicable to thin film retardation plate ${ }^{7}$. Figures 10 shows variation of refractive indexes of obliquely deposited $\mathrm{Ta}_{2} \mathrm{O}_{5}$ films and $\mathrm{WO}_{3}$ films around the wavelength of $0.6 \mu \mathrm{m}$ obtained from interference oscillation in spectral transmission data at normal incidence. In this region of refractive index value, reflective index is almost in linear relation with density according to Lorents-Lorenz fomula. Figs.10 teach that density decreases rapidly around $50^{\circ}-70^{\circ}$. Figures 11 show variations of the difference of refractive indices $\Delta \mathbf{n}$ between the normally incident polarized lights with its polarization direction in plane of vapor incidence and with its polarization direction normal to the vapor incidence in the case of $\mathrm{Ta}_{2} \mathrm{O}_{5}, \mathrm{WO}_{3}$, and $\mathrm{Bi}_{2} \mathrm{O}_{3}$. Irrespective of materials, $\Delta \mathrm{n}$ takes its maximamu around $\delta=70^{\circ}$. Figure 12 (a) shows $\Delta \mathbf{n}$ of different oxides obliquely deposited at $\delta=70^{\circ}$. Irrespective of the refractive indices of their crystalline states, oxides of heavy metals showed larger $\Delta \mathrm{n}$ since this birefringence is caused by the difference of refractive indices of metal oxides and voids. Fig.12 (b) shows slight decrease in $\Delta \mathrm{n}$ with increase in the substrate temperature during the oblique deposition of $\mathrm{WO}_{3}$ at $\delta=70^{\circ}$. 

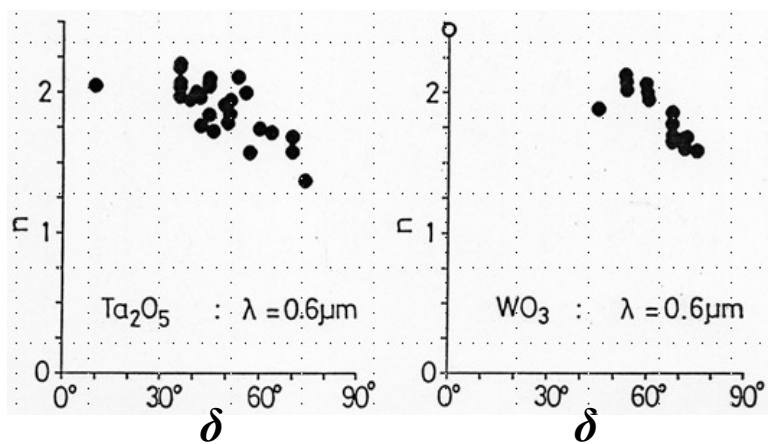

Figure 10 Plots of refractive indices at the wavelength $0.6 \mu \mathrm{m}$ against $\boldsymbol{\delta}$ in the case of obliquely deposited $\mathrm{Ta}_{2} \mathrm{O}_{5}$ film and $\mathrm{WO}_{3}$ film at ro
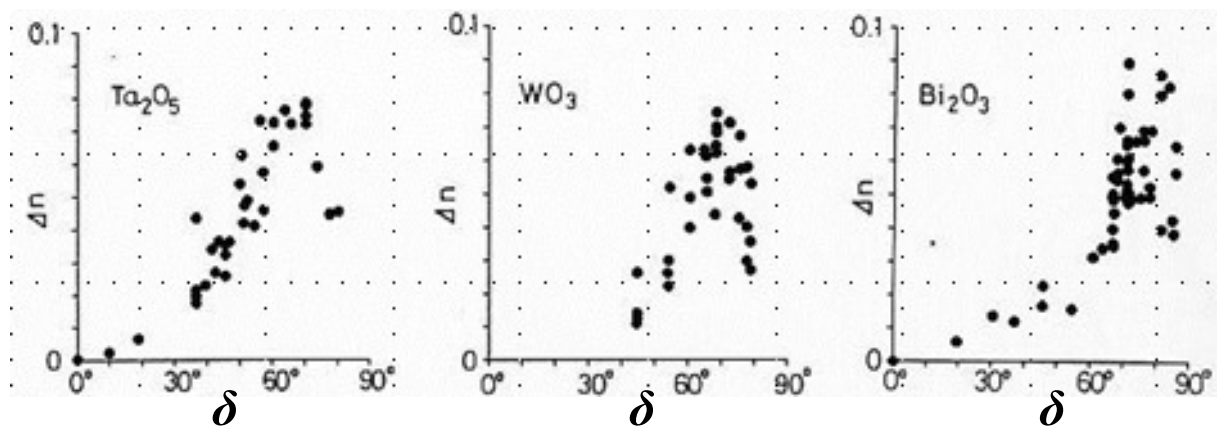

Figure 11 Plots of $\Delta \mathrm{n}$ against $\boldsymbol{\delta}$ in the case of obliquely deposited $\mathrm{Ta}_{2} \mathrm{O}_{5}$ film, $\mathrm{WO}_{3}$ film, and $\mathrm{Bi}_{2} \mathrm{O}_{3}$ film at

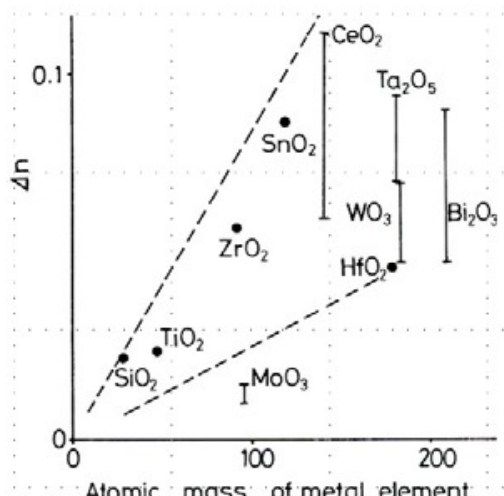

(a)

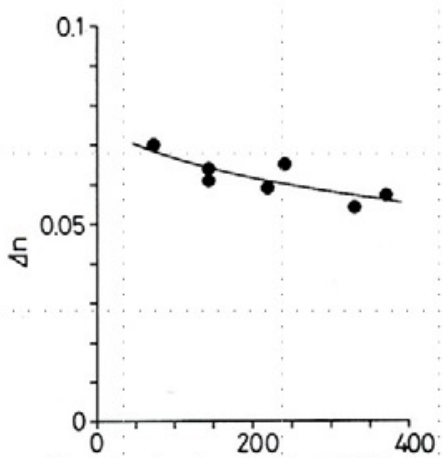

(b)

Figure 12 Plots of $\Delta \mathrm{n}$ (a) of various oxide thin films obliquely deposited at $\delta=70^{\circ}$ (b) as a function of the substrate temperature during the oblique deposition of $\mathrm{WO}_{3}$ at $\delta=70^{\circ}$.

This might be caused by enhancement of mobility of adatoms after the deposition by substrate heating in contrast to the frozen model in Fig.4. However, this relaxation of biaxial anisotropic nanostructure is small as shown in the value of decrease in $\Delta \mathrm{n}$ in Fig.12(b). Figure 13 shows two-storied obliquely deposited $\mathrm{Ta}_{2} \mathrm{O}_{5}$ films for application to thin film quarter wave plates. The sample (a) deposited in the vacuum of $0.004 \mathrm{~Pa}$ at the rate of $2.5 \mathrm{~nm} / \mathrm{sec}$ was transparent with a fine flat surface but colored brown because of oxygen deficiency, while the sample (c) deposited in the oxygen partial pressure of $0.07 \mathrm{~Pa}$ was not colored but translucent hazy film with a rugged surface influenced by the columnar structure. The sample (a), however, gradually lost its color in several days keeping its transparency without haze. We could make a homogeneous thin film quater wave plate on a $60 \mathrm{~mm} \times 250 \mathrm{~mm}$ glass substrate. Figure 14 shows a magnified view of a fractured cross-section of the sample (a) composed of fibrous structure thinner than $10 \mathrm{~nm}$. At the cross point of the two fractured cross-section of the sample (a) composed of fibrous structure thinner than $10 \mathrm{~nm}$. At the cross point of the black arrows, a plate-like structure can be observed which might be plucked out and fell when the sample was fractured 

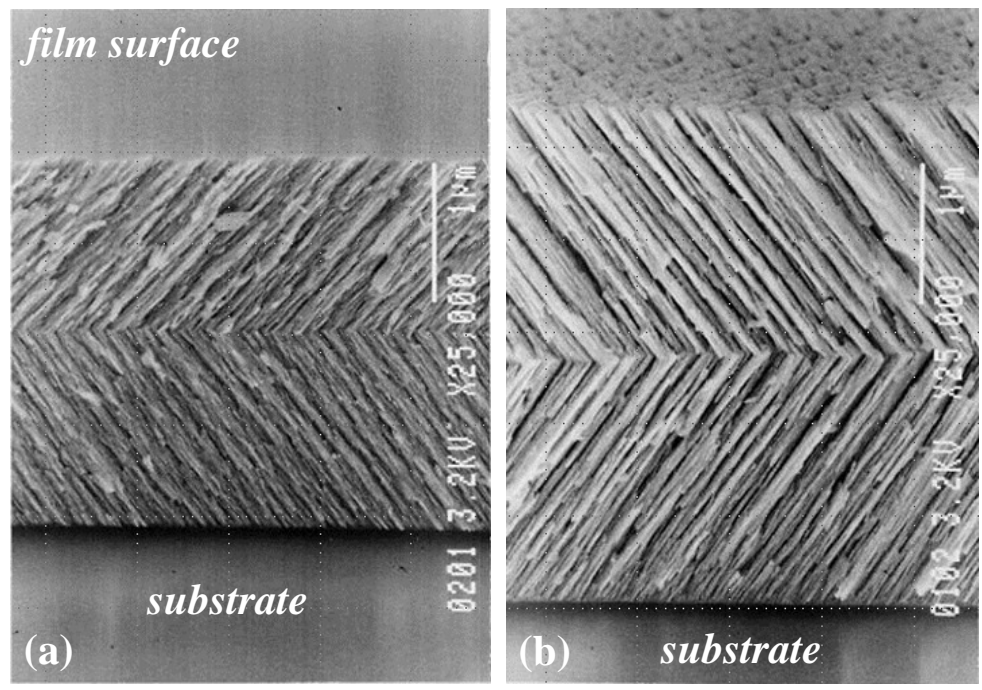

Figure 13 Obliquely deposited $\mathrm{Ta}_{2} \mathrm{O}_{5}$ films composed of two layers deposited opposite azimuthal direction to compensate asymmetric birefringence around the substrate normal. (a) a brown transparent sample deposited in the vacuum of $0.004 \mathrm{~Pa}$ and (b) a white translucent hazy sample deposited in the oxygen partial pressure of $0.07 \mathrm{~Pa}$.

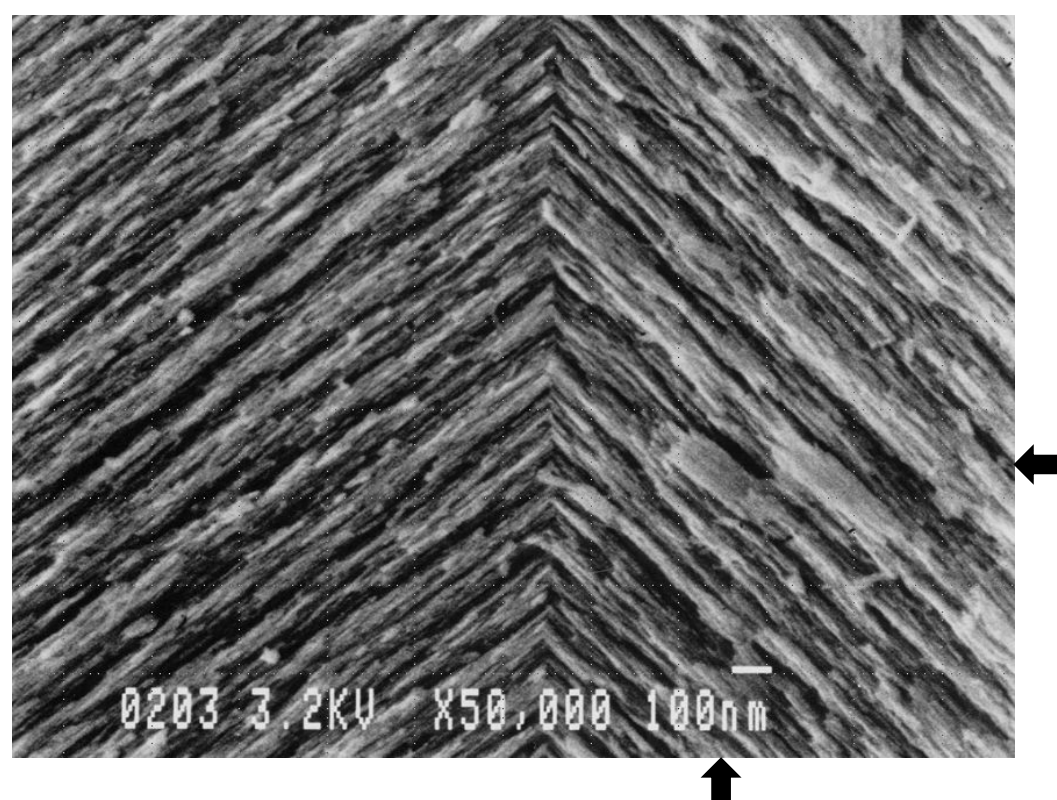

Figure 14 Magnified view of a fractured cross-section of the sample (a) composed of fibrous structure thinner than 10 $\mathrm{nm}$. At the cross point of the two black arrows, a plate-like structure can be observed which might be plucked out and fell over when the sample was fractured.

This indicate the obliquely deposited films in the sample (a) is a kind of quasi-multilayered structure composed of platelike structures with their thickness less than $10 \mathrm{~nm}$ as indicated in Fig.5 and 6.

\subsection{Nanometer-scale composite structures formed by simultaneous oblique sputter-deposition of two materials from opposite azimuthal directions}

Figure 15 shows a result of three dimensional computer modeling of self-shadowing by oblique deposition similar to Fig.2, but this time, two different particles are deposited in the same fluxes simultaneously from two opposite azimuthal directions at $\delta=60^{\circ}$, i.e. thick circles from the upper-left direction and thin circles from the upper-right direction ${ }^{10}$. The 


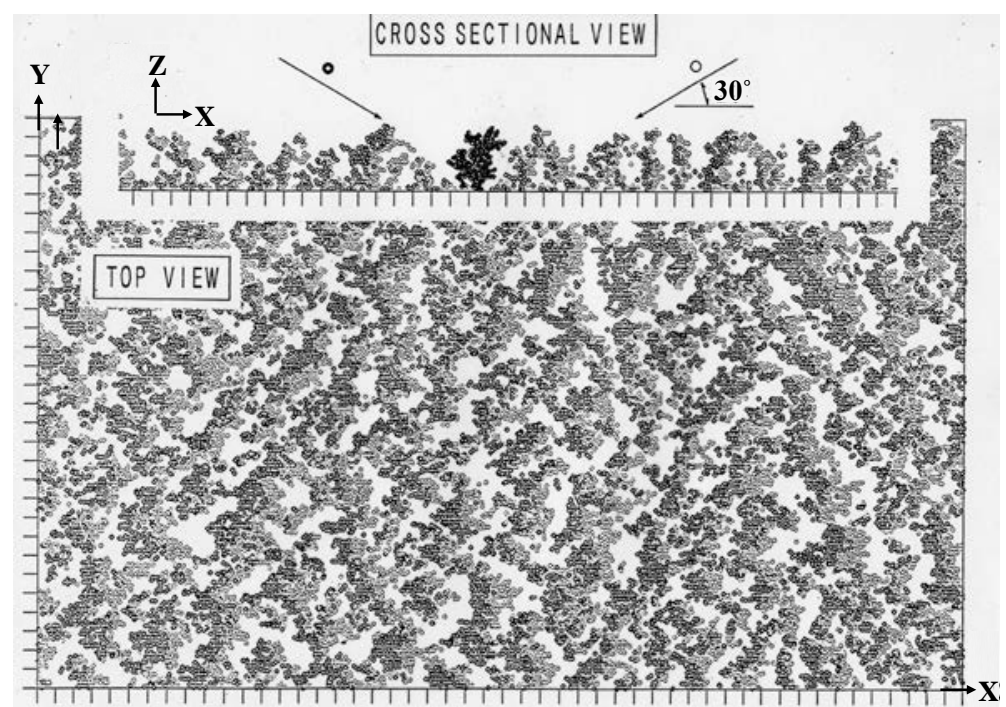

Figure 15 A result of three dimensional computer modeling of self-shadowing by simultaneous oblique deposition of two different particles: thick particles from the upper-left direction and thin particles from the upper-right direction at $\delta=60^{\circ}$ in the same fluxes.

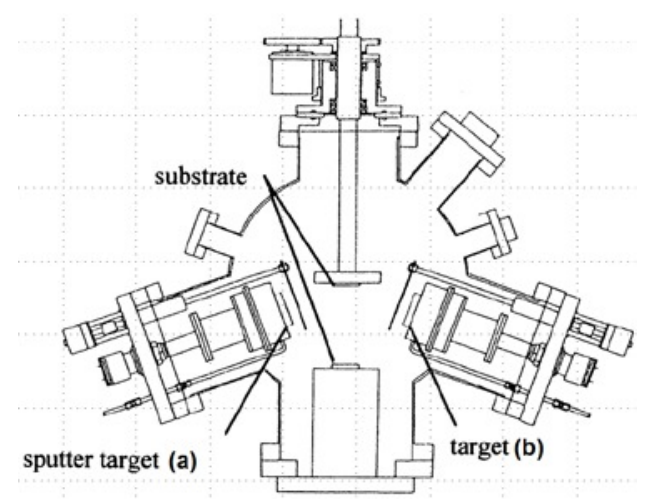

Figure16 Schematic drawing of a specially designed co-sputtering apparatus to realize simultaneous oblique deposition from two opposite azimuthal directions

cross sectional view shows the columns are not inclined but have grown normal to the substrate. The top view shows each cluster of thick circles and that of thin circles are placed back to back, forming quasi-periodic structure of a period of /thick circles' cluster/thin circles'cluster/voids/ along x-direction. Those clusters are less closely connected along the $\mathrm{x}$-direction than $\mathrm{y}$-direction forming quasi-periodic anisotropic nano-composite structure. Figure 16 shows a shematic drawing of a specially designed co-sputtering apparatus to realize simultaneous oblique deposition from two opposite azimuthal directions. Two 2-inch magnetron-type sputter-cathodes were employed. Using a $\mathrm{SiO}_{2}$ target and a $\mathrm{ZnTe}$ target, simultaneous oblique deposition was performed with a nominal volume fraction: $\mathrm{ZnTe}: \mathrm{SiO}_{2}=2: 3$. Figure 17 shows TEM images of the resultant anisotropic nanocomposite film removed from a $\mathrm{KCl}$ substrate. White arrows indicate the azimuthal directions of deposition for $\mathrm{SiO}_{2}$ and for $\mathrm{ZnTe}$, respectively. The bright portions correspond to $\mathrm{SiO}_{2}$ and dark portions corresponds to ZnTe. In the left TEM image, fringes can be observed in the dark portions, indicating that dark portions are composed of $\mathrm{ZnTe}$ crystalline nanoparticles. From the right image, the anisotropic $\mathrm{SiO}_{2}$ $\mathrm{ZnTe}$ nanocomposite can be assumed to be a vertical quasi multilayers of $\mathrm{SiO}_{2}$ and $\mathrm{ZnTe}$, or, a vertical quasi superlattice considering that the size of the period of $\mathrm{ZnTe} / \mathrm{SiO}_{2}$ is around $10 \mathrm{~nm}$, which is schematically drawn in Fig.18. Although there are various elaborate method to form vertical super-lattices, most of them can form the vertical superlattice in microscopic area. The simultaneous oblique deposition from two opposite azimuthal directions presented here may be one of few methods to form a thin film with properties of vertical superlattices in a large area even of sub squaremeter. 

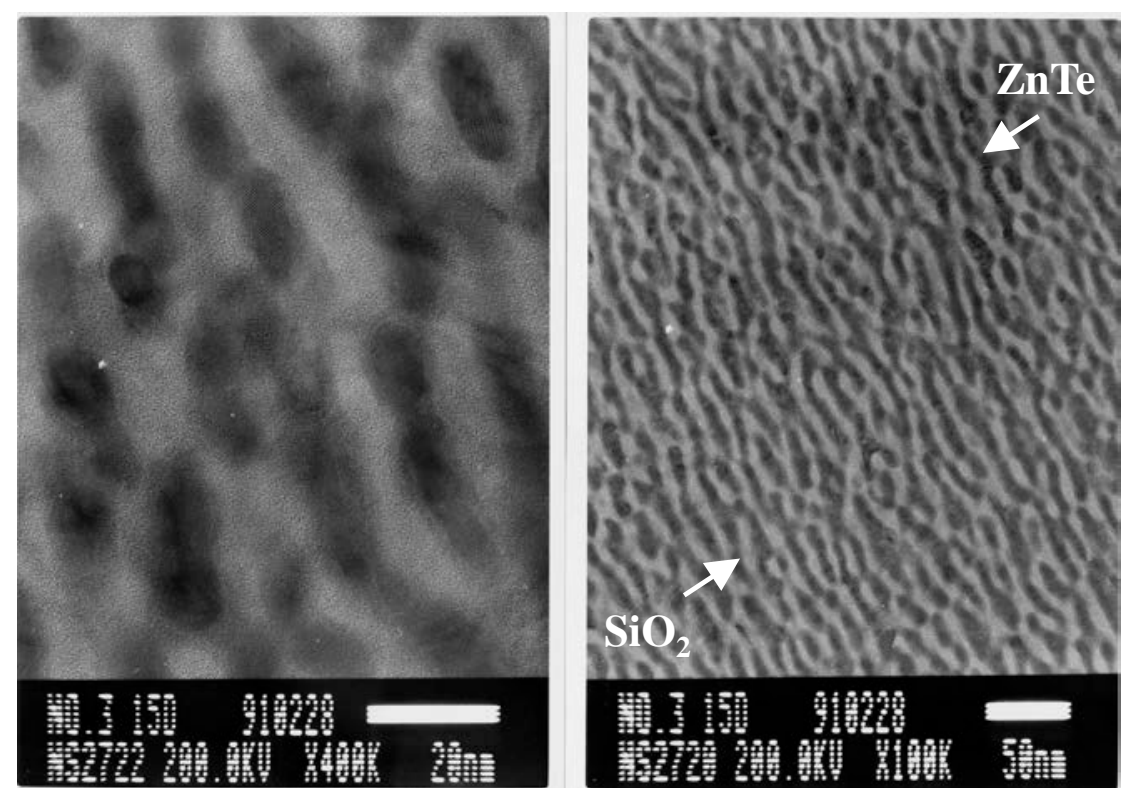

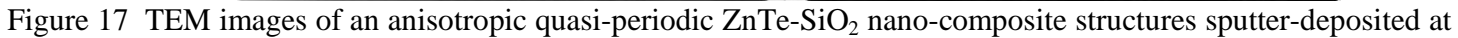
room temperature on a $\mathrm{KCl}$ crystal substrate using theapparatus shown in Fig.16.

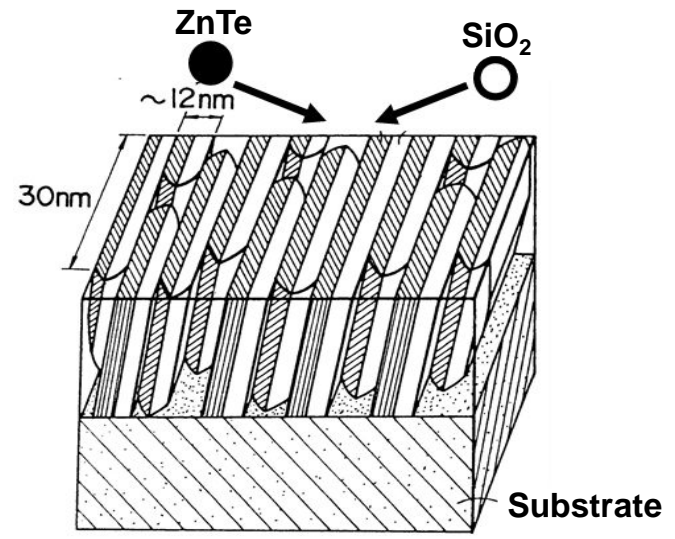

Figure 18 Schematic drawing of a vertical quasi superlattice by simultaneous oblique deposition of $\mathrm{ZnTe}_{\text {and }} \mathrm{SiO}_{2}$. The inclination of the structure reflect the volume ratio of $\mathrm{ZnTe}: \mathrm{SiO}_{2}=2: 3$, and can be changed by changing the volume ratio

\section{SELF-SHADOWING IN ANODIZED POROUS SILICON}

The visible photoluminescence from porous Si reported Canham ${ }^{11}$ in 1990 had a great impact and stirred up related researches because of its intense photoemission observable with the naked eye and easy reproducibility. The topic contained the basic interest why silicon with an electronic structure of indirect transition could give such high efficiency photoluminescence specially in connection with quantum effect in nanostructured material, and also contained the interest of possibility to open doors to Si opt-electronics. Figures 19 (1) shows a photograph of the porous Si showing photoluminescence under the illumination of $4 \mathrm{~W}$ portable black light reproduced in our laboratory in $1991^{12}$. Fig.19(2) shows a photograph of the same sample showing photoluminescence under the same black light in 2018 after 27 year's storage in the air in the room temperature, showing high durability of the system especially for oxidation in the normal atmosphere. Although the colors, i.e. the emission spectra of luminescence were different between the samples depending on the anodization conditions, excitation spectra showed that the photoluminescence took place when the photon energy for excitation exceeds $3 \mathrm{eV}$ which corresponded to photo absorption by direct transition of bulk Si without 
quantum confinement effect ${ }^{12,13}$. On the other hand, the photon energy of the photoemission was variable depending on the anodization conditions. Therefore, it was suggested that photoexcitation took place in the remaining bulky portion in porous silicon and the photo-excited electrons were transmitted to peripheral portions with preferable electronic structure for photoemission formed by electrochemical interaction between silicon and hydrofluoric acid (HF) ${ }^{14}$. However, the mechanism of intense photoluminescence of porous silicon had been controversial throughout 1990s.

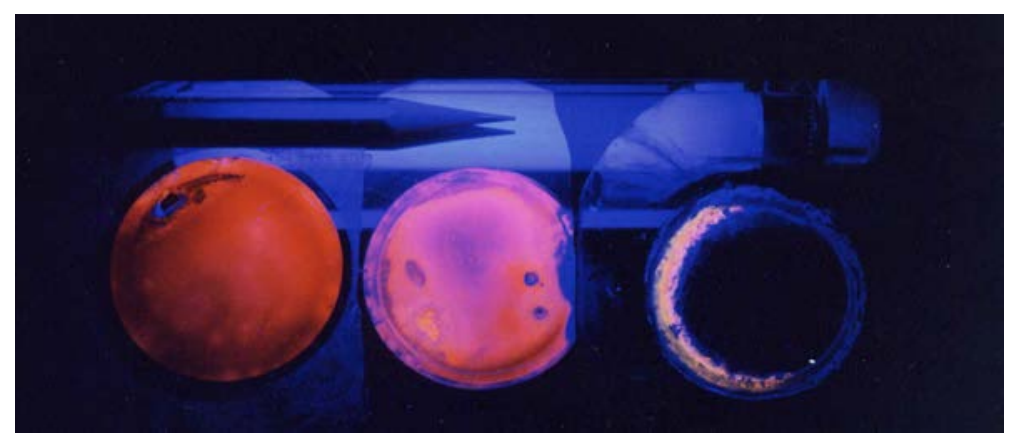

(1) taken in 1991 (a)

(b)

(c)

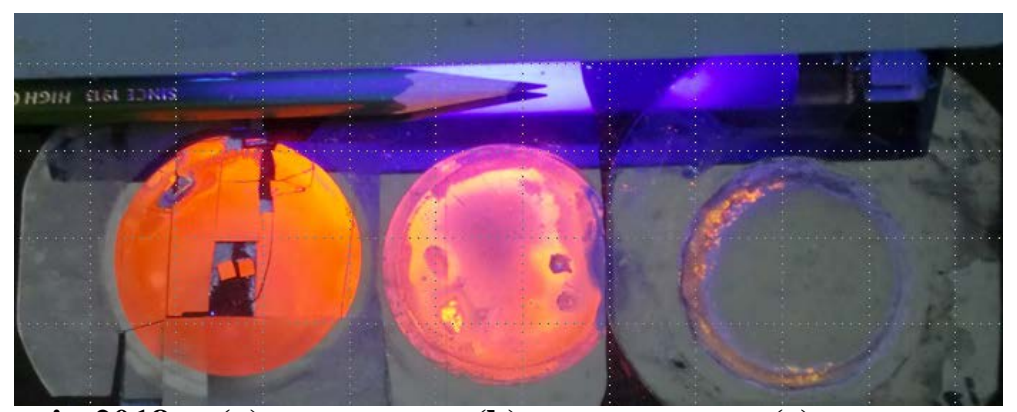

(b) taken in 2018 (a)

(b)

(c)

Figure 19 (1) Photograph of the three samples luminous in (a) orange, (b) pink and (c) partly greenish yellow and partially pink under illumination by a 4-watt portable black light, taken in 1991. The pinnacle shape is a pencil placed for reference of size. (2) Photograph of the same sample under the illumination of the same lamp taken in 2018.

Anodizaition in HF-based solution can make Si nanoporous ( pore width $<2 \mathrm{~nm}$ ) or macroporous (pore width $>50 \mathrm{~nm}$ ) depending on substrate resistivity and anodization conditions ${ }^{11}$. The geometry of porous Si was looked as existence of extremely small holes running orthogonal to the Si surface. Depending on the size of pores, different mechanism of anodization has been proposed. In the case of extremely microporous porous Si, the geometry is more like fractal than Euclidean. Here, we follow one of the proposed mechanism. Since HF cannot corrode Si, pore formation by dissolution of $\mathrm{Si}$ in anodization is attained by supply of holes. Figure 20 shows a result of a two dimensional simulation of the process of porous Si layer formation, in which holes supplied by anode are supposed to make diffusion, i.e. random walks with drift towards the Si-HF interface. When a hole arrived at the Si-HF interface, Si corroded into electrolyte as $\mathrm{SiF}_{4}$ and eliminated away remaining pore filled with $\mathrm{HF}$ to make new Si-HF interface. The black dendrite patterns in Fig.20 are those accumulated pores where Si was corroded and filled with HF. Therefore, the black dendrite patterns are traces of accumulation of arrivals of holes at Si/HF interfaces, or "deposition” of holes. Since extended dendrites, i.e. $\mathrm{Si} / \mathrm{HF}$ interface easily capture successively coming holes, they prevent holes from travelling deep into porous Si layer. Therefore, Si crystallites are remained between the dendrite pores in the porous Si layer. This is self-shadowing of holes similar to the self-shadowing in oblique deposition. However since mean free path of holes in Si is short enough in comparison to the system size, holes travel in the manner of drifted diffusion in contrast to the ballistic deposition in oblique deposition in vacuum. Since the growth of the dendrites in Fig.20 attained by drift diffusion is a variation of diffusion limited aggregation, the resultant patterns in the porous Si layer is of Fractal nature. 


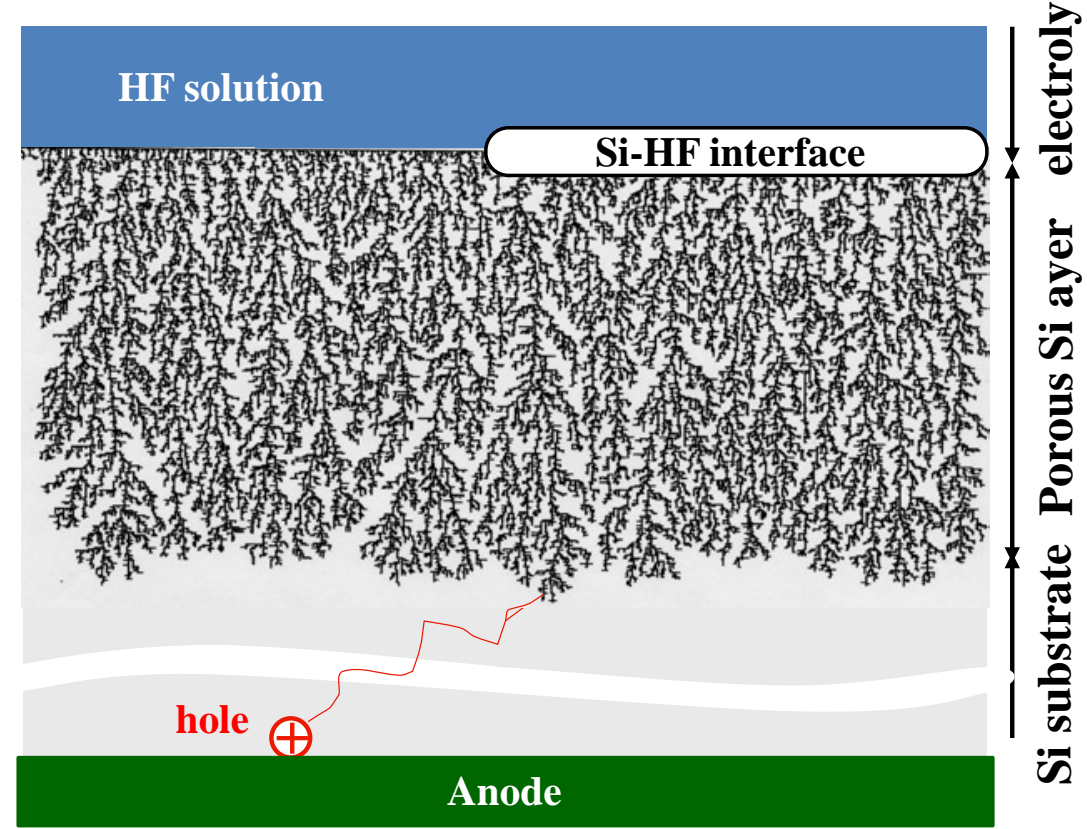

Figure 20. Result of a two dimensional simulation of the process of porous Si layer formation, in which holes supplied by anode are supposed to make drift diffusion, i.e. random walks with drift towards the Si-HF interface.

\section{SUMMARY}

K. L. Chopra, the author of "Thin film phenomena" (McGraw-Hill, New York and London, 1969 ) stated that nanotechnology should be accompanied by the ability to make nanostructures in large scale or in large area ${ }^{15}$. In this sense, self-shadowing is one of the key phenomena to be utilized although it appeared far before the nanotechnologies got active in late 1990s. Starting with a topic of spontaneous formation and evolution of ripple structures by oblique Ar ion beam bombardment of the surface of transparent fused silica, methods of nanostructure formation in large area with simple macroscopic experimental condition setting was revived. Here, self-shadowing effect was spotlighted as a simple means without "nanotechnology" to realize nanostructured thin films and layers with two typical prominent nanostructure effects which are both observable with the naked eye, that is, birefringence of obliquely deposited thin films and intense visible photoluminescence of porous Si. In oblique deposition, the self-shadowing effect takes place in ballistic deposition and accumulation of materials on a vacuum / substrate interface. In porous Si, the drift diffusion of positive holes from an anode thorough Si to Si / HF solution interface causes a modified diffusion limited aggregation of positive holes which leads directly to formation of pores. Thin film quarter wave plate utilizing the birefringence of obliquely deposited $\mathrm{Ta}_{2} \mathrm{O}_{5}$ film could be homogeneously formed on $60 \mathrm{~mm} \times 250 \mathrm{~mm}$ glass substrate and visible photoluminescent porous Si could be formed on throughout the Si wafer of $101.6 \mathrm{~mm}$ in diameter. The former one is still clear and transparent and the latter one is still highly photoluminescent even now after 27 or more years irrespective of their origin of remarkable properties based on nanostructures. 


\section{REFERENCES}

[1] Motohiro, T., "Characteristic erosion of silica by oblique argon ion beam bombardment,” Thin Solid Films 147, 153-165 (1987).

[2] Motohiro, T., "Spontaneously emergent ripples-pattern formation by ion beam sputter-etching and wind," Forma 26, 7-10(2011). <http://www.scipress.org/journals/forma/frame/26.html> (20 July 2018)

[3] Dirks, A. G. and H. J. Leamy, "Columnar microstructure in vapor-deposited thin films,"Thin Solid Films 47(3), 219-233 (1977).

[4] Leamy, H. J., Gilmer, G. H. and Dirks, A. G., "The Microstructure of Vapor Deposited Thin Films," in Kaldis X. X. [Current Topics in Materials Science Vol.6], North-Holland, Amstredam, 309-343 (1980).

[5] Keitoku S. and Nishioka K.," Grain Structure and Magnetic Anisotropy of Fe Film Evaporated Obliquely from Two Sources,” Jpn. J. Appl. Phys. 20(7), 1249-1253 (1981).

[6] Motohiro T., Noda, S., Isogai A. and Kamigaito O. "Modeling of Nanophase Connectivity in Substance-Void Composite by Oblique Deposition”, Mat. Res. Soc. Symp. Proc. Vol. 159, 419-424 (1990).

[7] Motohiro T. and Taga, Y., "Thin film retardation plate by oblique deposition," Applied Optics 28(3), 24662482 (1989).

[8] Motohiro, T. and Taga, Y., "Elliptical blue patches growing in obliquely deposited $\mathrm{WO}_{3}$ films," Thin Solid Films 172, L71-L74 (1989).

[9] Macleod, H. A., "Structure-related optical properties of thin films," J. Vac. Sci. Technol. A4, 418-422 (1986).

[10] Motohiro, T., Takeda, Y., Watanabe, Y. and Noda, S., "Nanometer-Scale Composite Structures Formed by Simultaneous Oblique Sputter-Deposition od Two Materials from Different Directions,” Mat. Res. Soc. Symp. Proc. Vol. 206, 423-428 (1990).

[11] Canham L. T., "Silicon quantum wire array fabrication by electrochemical and chemical dissolution of wafers," 57, 1046-1048 (1990).

[12] Motohiro, T., Kachi T., Miura F., Takeda Y., Hyo-do, S. and Noda, S., "Excitation spectra of the visible photoluminescence of anodized porous silicon,” Jpn. J. Appl. Phys. 31 Part 2 (3A), L207-L209 (1992).

[13] Motohiro, T., Kachi T., Miura F., Takeda Y., Hyo-do, S. and Noda, S., "Excitation spectra of the visible photoluminescence of anodized porous silicon,” Mat. Res. Soc. Symp.Proc. 256, 53-58(1992).

[14] Motohiro, T., Takeda Y., Hyo-do, S., Hioki T. and Noda, S., "An origosilane bridge model: nanocomposite nature of the rigin of the intense visible photoluminescence of porous silicon," [Porous Silicon], World Scientific, Singapore, New Jergy, London and Hong Kong, 99-131 (1994).

[15] Chopra, K. L. private communication 